\title{
Circulating microRNA profiles and the identification of miR-593 and miR-511 which directly target the PROP1 gene in children with combined pituitary hormone deficiency
}

\author{
YANYAN HU ${ }^{1}$, QIAN WANG ${ }^{1}$, ZENGMIN WANG $^{1}$, FENGXUE WANG $^{1}$, XIAOBO GUO $^{2}$ and GUIMEI LI ${ }^{1}$ \\ Departments of ${ }^{1}$ Pediatrics and ${ }^{2}$ Gastrointestinal Surgery, Shandong Provincial Hospital \\ Affiliated to Shandong University, Jinan, Shandong 250021, P.R. China
}

Received August 22, 2014; Accepted November 26, 2014

DOI: $10.3892 /$ ijmm.2014.2016

\begin{abstract}
Since the tissue of children with combined pituitary hormone deficiency (CPHD) is not readily accessible, a new focus in children with CPHD is the blood-based expression profiling of non-protein coding genes, such as microRNAs (miRNAs or miRs), which regulate gene expression by inhibiting the translation of mRNAs. In this study, to address this, we identified potential miRNA signatures for CPHD by comparing genome-wide miRNA expression profiles in the serum of children with CPHD vs. normal (healthy) controls. Human embryonic kidney $293 \mathrm{~T}$ cells were transfected with miR-593 or miR-511 oligonucleotides. Potential target gene expression was validated by western blot analysis for proteins and by miR-593 or miR-511 reporter assay using PROP1 gene 3'-untranslated region (3'-UTR) reporter. The miR-593 and miR-511 levels in the serum of 103 children with CPHD were assessed using the reverse transcription-quantitative polymerase chain reaction (RT-qPCR) method. We found 23 upregulated and 19 downregulated miRNAs with abnormal expression in children with CPHD compared with the normal controls using miRNA microarray analysis and RT-qPCR. miR-593 and miR-511 targeted the 3'-UTR of the PROPI gene and attenuated the expression of PROP1. The levels of miR-593 and miR-511 in the serum of children with CPHD were increased compared with those in the control subjects. According to Youden's index, the sensitivity was 82.54 and $84.86 \%$, and the specificity was 98.15 and $91.36 \%$ for miR-593 and miR-511, respectively. The
\end{abstract}

Correspondence to: Dr Guimei Li, Department of Pediatrics, Shandong Provincial Hospital Affiliated to Shandong University, No. 324 Jingwu Road, Jinan, Shandong 250021, P.R. China E-mail:1gmusa05@gmail.com

Dr Xiaobo Guo, Department of Gastrointestinal Surgery, Shandong Provincial Hospital Affiliated to Shandong University, No. 324 Jingwu Road, Jinan, Shandong 250021, P.R. China

E-mail: guo992352@hotmail.com

Key words: microRNAs, PROP1 gene, combined pituitary hormone deficiency, microarray various levels of specific miRNAs, particularly miR-593 and miR-511 whose direct target is the PROPI gene, may serve as a non-invasive diagnostic biomarkers for children with CPHD.

\section{Introduction}

Great progress has been made in our understanding of the development of the anterior pituitary gland and of the mechanisms that underlie the diagnosis of combined pituitary hormone deficiency (CPHD). Naturally occurring and transgenic murine models have demonstrated a role for many of these molecules in the etiology of CPHD $(1,2)$. Anatomical abnormalities in the pituitary gland may be associated with other midline abnormalities and variable endocrine disorders, ranging from isolated growth hormone deficiency (IGHD) to CPHD $(3,4)$. CPHD is a severe endocrine disorder in children. Different types and severities of hormonal deficiencies with various clinical manifestations are observed in children with CPHD. The definite diagnosis of CPHD is necessary. Pituitary magnetic resonance imaging (MRI) and hormones are essential examinations for the diagnosis of CPHD. Significant advances in molecular biology and the normal development of the pituitary gland have led to a greater understanding of the genetic basis of CPHD and related conditions.

PROPl has been mapped to chromosome $5 \mathrm{q}$ and encodes a protein of 226 amino acids. The DNA-binding homeodomain consists of $3 \alpha$-helical regions and the majority of mutations reported to date affect this region. PROPI is essential for the differentiation of gonadotrophs in fetal life. The spectrum of gonadotropin deficiency is again extremely variable, ranging from hypogonadism and the lack of puberty to spontaneous pubertal development and infertility $(5,6)$. However, it is has been suggested that PROPI is not required for gonadotroph determination, but is required for differentiation. A 2-bp deletion (delA301, G302) is now believed to be a mutational 'hot spot' within PROPI (7-9). To date, mutations in PROPI are associated with growth hormone $(\mathrm{GH})$, thyrotropin (TSH), prolactin (PRL) and gonadotropin deficiencies. Fifteen distinct recessive mutations have been identified in approximately 147 individuals from 76 to 84 pedigrees originating in 20 different countries, suggesting that mutations within PROPI are the most common genetic cause of CPHD, with 
incidence rates quoted between 50 and $100 \%$ in familial cases of CPHD (10-12).

Recently, researchers have found a new class of short, endogenously non-coding RNAs termed microRNAs (miRNAs or miRs) in animals and plants (13-15). It is now clear that they play pivotal roles in a wide array of biological processes, including differentiation and cell proliferation and apoptosis $(16,17)$. They regulate the expression of protein-coding genes by degrading or inhibiting the translation of the targeted mRNAs (18). Emerging evidence strongly suggests that abnormal miRNA expression is a common and important characteristic of human diseases $(19,20)$. To date, a number of studies have proven that a non-invasive approach for the circulating blood-based miRNA identification of biomarkers is extremely valuable and useful in diseases (17,19-21).

miRNA profiling using microarray technology has recently been developed and applied to the study of a variety of conditions $(22,23)$. Based on these studies, we can now perform blood-based miRNA profiling to search for CPHD. In this study, to ascertain whether circulating miRNA expression signatures can distinguish children with CPHD from normal (healthy) controls, we performed genome-wide miRNA expression profiling from serum samples in children with CPHD and healthy controls. Using expression profile data and data from reverse transcription-quantitative PCR (RT-qPCR), our study indicates that the various levels of specific miRNAs, particularly miR-593 and miR-511 whose direct target is the PROPI gene, may serve as non-invasive diagnostic biomarkers for children with CPHD.

\section{Materials and methods}

Blood sample collection. A total of 206 participants at the Department of Pediatrics of Shandong Provincial Hospital Affiliated to Shandong University (Jinan, China) between 2009 and 2013 were recruited in this study. This included 103 children with CPHD ( 88 boys and 15 girls; age, 11.6 \pm 3.5 years; range, 8.2-16.6 years) and 103 normal (healthy) controls (85 boys and 18 girls; age, $11.2 \pm 3.8$ years; range, $7.5-16.0$ years). There were no significant differences in the age and gender between the CPHD group and the control group $(\mathrm{P}>0.05)$. All children had at least one anterior pituitary hormone deficiency in addition to GHD, and were therefore diagnosed as having CPHD. Whole blood samples $(4 \mathrm{ml})$ were collected from the children with CPHD and the normal controls into K2-EDTA-coated tubes. Subsequently, $3 \mathrm{ml}$ of the whole blood was centrifuged at $1,000 \mathrm{x} \mathrm{g}$ for $10 \mathrm{~min}$, and then the serum (approximately $1 \mathrm{ml}$ ) was aliquoted into an RNase-free tube. All the serum samples were stored at $-80^{\circ} \mathrm{C}$ prior to RNA extraction. The study was approved by the institutional review board of the hospital. Written informed consent was obtained from the parents of all the subjects.

miRNA microarray. Total RNA from 7 children with CPHD and 7 normal controls was isolated using TRIzol reagent (Invitrogen, Carlsbad, CA, USA) and the miRNeasy Mini kit (Qiagen, Hilden, Germany) according to manufacturer's instructions. For each sample that passed RNA quantity measurement using the NanoDrop 1000 spectrophotometer (Thermo Fisher Scientific, Waltham, MA, USA) $1 \mu \mathrm{g}$ of total RNA was 3'-end-labeled with $\mathrm{Hy}^{\mathrm{TM}}$ fluorescent label using the miRCURY ${ }^{\mathrm{TM}} \mathrm{Hy} 3^{\mathrm{TM}} / \mathrm{Hy}^{\mathrm{TM}}$ Power Labeling kit (Exiqon, Vedbaek, Denmark), and hybridized to miRCURY ${ }^{\mathrm{TM}}$ LNA Arrays (version 18.0), according to the manufacturer's instructions. The seventh generation of miRCURY ${ }^{\mathrm{TM}}$ LNA Arrays (version 18.0) (Exiqon) contains 3,100 capture probes, covering all human, mouse and rat miRNAs annotated in miRBase 18.0. In addition, this array contains capture probes for 25 miRPlus $^{\mathrm{TM}}$ human miRNAs. Following hybridization, the slides were washed several times using the Wash buffer kit (Exiqon), and dried by centrifugation for $5 \mathrm{~min}$ at $400 \mathrm{rpm}$. The slides were scanned using the Axon GenePix 4000B microarray scanner (Axon Instruments, Foster City, CA, USA). Scanned images were imported into GenePix Pro 6.0 software (Axon Instruments) for grid alignment and data extraction. The data were normalized using median normalization. Following normalization, differentially expressed miRNAs were identified through fold change filtering. Hierarchical clustering was performed using MEV software (version 4.6, TIGR).

Bioinformatics: sequence analysis. We researched microRNAs that were associated with children with CPHD through the miRBase (http://microrna.sanger.ac.uk/). Putative targets were identified using the microrna.org (http://www.microrna.org/ microrna/home.do), TargetScan (http://www.targetscan.org/ vert_40/) and RNAhybrid databases (http://bibiserv.techfak. uni-bielefeld.de/rnahybrid/submission.html).

Cell lines, culture and transfection. The human embryonic kidney (HEK)293T cells were preserved at our institute and cultured in Dulbecco's modified Eagle's medium (DMEM; Sigma, St. Louis, MO, USA) supplemented with $10 \%$ fetal calf serum (FCS). Exponentially growing cells were used for the experiments.

Stability-enhanced miRNAs, stability-inhibited miRNAs and negative control RNA-oligonucleotides were obtained from Ambion Inc. (Austin, TX, USA). The day prior to transfection, the HEK293T cells were seeded in antibiotic-free medium. The transfection of miRNAs was carried out using Lipofectamine 2000 in accordance with the manufacturer's instructions (Invitrogen). Stability-enhanced miRNAs, stabilityinhibited miRNAs and negative control RNA-oligonucleotides were transfected at a final concentration of $50 \mathrm{nM}$ unless otherwise indicated. The level of miR-593 and miR-511 expression in the transfected HEK293T cells was assayed by RT-qPCR $48 \mathrm{~h}$ after transfection as described below.

$R T-q P C R$. Total RNA from was extracted from the serum samples and cultured cells using a miRNeasy Mini kit (Qiagen) designed to isolate small molecular weight nucleic acids. The concentration and purity of the total RNA samples were measured using the SmartSpec Plus spectrophotometer (BioRad, Hercules, CA, USA). The ratio of A260:A280 was used to indicate the purity of total RNA.

cDNA was generated using the miScript Reverse Transcription (RT) kit (Qiagen). According to the manufacturer's instructions, $1 \mu \mathrm{g}$ total RNA, $1 \mu \mathrm{l}$ miScript reverse transcriptase mix, $4 \mu \mathrm{l}$ 5X miScript RT buffer and appropriate volume RNase-free water were mixed well and incubated for $60 \mathrm{~min}$ at $37^{\circ} \mathrm{C}$, and then incubated for $5 \mathrm{~min}$ at $95^{\circ} \mathrm{C}$ to inactivate miScript 
reverse transcriptase mix. All reverse transcription procedures and no-template controls were run at the same time.

A miScript SYBR-Green PCR kit (Qiagen) was used to measure the expression of mature miR-593 and miR-511 in the samples and cells following reverse transcription. Quantitative (real-time) PCR was performed using on an Mx3005P qPCR System (Stratagene, La Jolla, CA, USA). Following the manufacturer's instructions, the $20 \mu \mathrm{l}$ PCR mixture included $2 \mu \mathrm{l}$ reverse transcription product, $10 \mu 12 \mathrm{X}$ QuantiTect SYBRGreen PCR Master Mix, $2 \mu 1$ 10X miScript Universal Primer, $2 \mu 1$ 10X miScript Primer Assay (for miR-593 and miR-511; Qiagen) and $4 \mu \mathrm{l}$ RNase-free water. The reaction mixtures were incubated at $95^{\circ} \mathrm{C}$ for $15 \mathrm{~min}$, followed by 40 amplification cycles of $94^{\circ} \mathrm{C}$ for $15 \mathrm{sec}, 55^{\circ} \mathrm{C}$ for $30 \mathrm{sec}$ and $70^{\circ} \mathrm{C}$ for $30 \mathrm{sec}$. We also quantified transcripts of U6 small nuclear RNA using the Hs_RNU6B_2 miScript Primer Assay (Qiagen) for normalizing the levels of miR-593 and miR-511.Hs_RNU6B_2 was used as an endogenous control. Each sample was analyzed 2 times. The threshold cycle $(\mathrm{Ct})$ was defined as the fractional cycle number at which the fluorescence exceeds the given threshold. The obtained data were translated into the $\log 2$ scale, as previously described (29). The $2^{-\Delta \Delta \mathrm{Ct}}$ method was used to analyze the relative expression of the miRNAs.

Western blot analysis. The HEK293T cells were transfected with miR-593 precursor, miR-511 precursor, miR-593 inhibitor, miR-511 inhibitor, or the negative control in 6-well plates. Following transfection, the cells were cultured for $48 \mathrm{~h}$, protein was extracted using mammalian protein extraction reagent (Pierce, Rockford, IL, USA) supplemented with protease inhibitors cocktail (Sigma). Protein samples $(50 \mu \mathrm{g})$ were resolved by $10 \%$ sodium dodecyl sulfate-polyacrylamide gel electrophoresis (SDS-PAGE) and then transferred onto PVDF membranes. The membranes were blocked with TBST buffer (TBS plus $0.1 \%$ Tween-20) containing 5\% w/v non-fat milk and hybridized with primary antibody (rabbit polyclonal antibody, ab94500), followed by incubation with specific HRP-conjugated secondary antibody (anti-rabbit IgG antibody, ab191866). Protein bands were visualized using the ECL detecting system (Amersham Biosciences, Uppsala, Sweden). Rabbit polyclonal anti-PROP1 (1:1,000; ab94500; Abcam, Cambridge, MA, USA) and anti-rabbit IgG antibody (1:1,000; ab191866; Abcam) were used. Monoclonal anti-GAPDH (1:5,000; ab181602; Abcam) was used as a loading control.

Luciferase activity assay. A 230-bp fragment of the wild-type PROP1 3'-untranslated region (3'-UTR) containing the putative miR-593 or miR-511 binding site was amplified by PCR using the following primers: forward, 5'-ACCAAGCTTGTACCA CCAAGGTGATCCC-3' and reverse, 5'-ACCACTAGTGCA GGCAGCTCCACCGAGGCATC-3'. A mutant 3'-UTR of PROPl was synthesized by PCR, whose sequence contained 5'-TCGTGAATATACAAGAAAATG-3' or 5'-GCTACTGG AAGAGACAG GGCAAG-3' (the letters in italic and bold font indicate nucleotides which are mutated) and cloned downstream of the luciferase gene in the pMIR-report luciferase vector (Ambion, Inc.). This construct, named PROP1-3'-UTR or PROP1-3'-UTR-Mut was used for the transfection of HEK293T cell lines. HEK293T cells were cultured in 24-well plates. In each well, 10 ng of Renilla luciferase, phRL-TK vector (Promega,
Madison, WI, USA) were co-transfected to normalize for transfection effciency. A total of $500 \mathrm{ng}$ of REPORT, PROPI3'-UTR or PROP1-3'-UTR-Mut together with $10 \mathrm{nM}$ miR-593 or miR-511 or the negative control was also co-transfected into the cells in 24-well plates. Transfection was carried out using Lipofectamine 2000 and Opti-MEM I reduced serum medium (Life Technologies, Carlsbad, CA, USA) in a final volume of $0.5 \mathrm{ml}$. The transfection of the same combinations of plasmid and RNAs as repeated 3 times. After $48 \mathrm{~h}$, the cells were harvested with $100 \mu \mathrm{l}$ PLB reagent (Promega) and $20 \mu \mathrm{l}$ cell lysates prepared in Reporter Lysis Buffer (Promega), Firefly luciferase activity was measured for each well using the Dual luciferase assay kit (Promega) with an analytical luminometer (TD-20/20; Turner Designs, Sunnyvale, CA, USA) according to the manufacturer's instructions. Briefly, a $10 \%$ volume of cell lysate $(20 \mu \mathrm{l})$ was added to $100 \mu \mathrm{l}$ of LAR II, and then the reaction was terminated by the addition of $100 \mu 1$ Stop and Glo ${ }^{\circledR}$ Reagent. Normalized relative luciferase activity (RLA) was calculated using the following formula: RLA $=$ (Firefly luciferase)/(Renilla luciferase).

Statistical analysis. The differences between groups were estimated using the Pearson $\chi^{2}$ test, Student's t-test and ANOVA test. Receiver operating characteristic (ROC) curves and the area under the curve (AUC) calculations were performed to determine the threshold value of the children with CPHD and the normal controls. SPSS 15.0 software (SPSS Inc., Chicago, IL, USA) was used for all statistical analyses, and a value of $\mathrm{P}<0.05$ was considered to indicate a statistically significant difference. Data are expressed as the means \pm standard deviation from at least 3 separate experiments.

\section{Results}

Global serum miRNA profiling and data analysis in children with CPHD. miRNA expression profiles from the serum samples of 7 children with CPHD and 7 normal controls were analyzed by microarray analysis. Hierarchical clustering analyses of the overall expression profile divided the samples into 2 groups: children with CPHD and normal (healthy) controls (Fig. 1). The threshold set for up- and downregulated miRNAs was a fold change $\geq 6.0$ and a $P$-value $\leq 0.05$. Our results revealed 23 upregulated and 19 downregulated miRNAs with abnormal expression levels in children with CPHD compared with the normal controls (Fig. 1 and Table I). The expression levels of miR17-5p, miR-593, miR-23a-5p, miR-586, miR-1180, miR-508-5p, miR-511, miR-646, miR-634, miR-149-5p, miR-24-3p, miR-1267, miR-504 and miR-1270 were upregulated (Fig. 2A) $(\mathrm{P}<0.05)$. However, the expression levels of miR-1225-5p, miR-1909-5p, miR-512-5p, miR-3927, miR-1203, miR-2110, miR-501-5p, miR-648, miR-4729, miR-4475, miR-1914-3p and miR-5002-5p were downregulated (Fig. $2 \mathrm{~B})(\mathrm{P}<0.05)$ in the children with CPHD compared with the normal controls.

Prediction and identification of the candidate miRNAs, miR-593 and miR-511, which directly target the PROP1 gene. Among the targets predicted by the microrna.org, TargetScan databases and RNAhybrid database online search programs, we identified the PROPl gene as a possible target of miR-593 and miR-511 (Fig. 3A). The results of the database search 


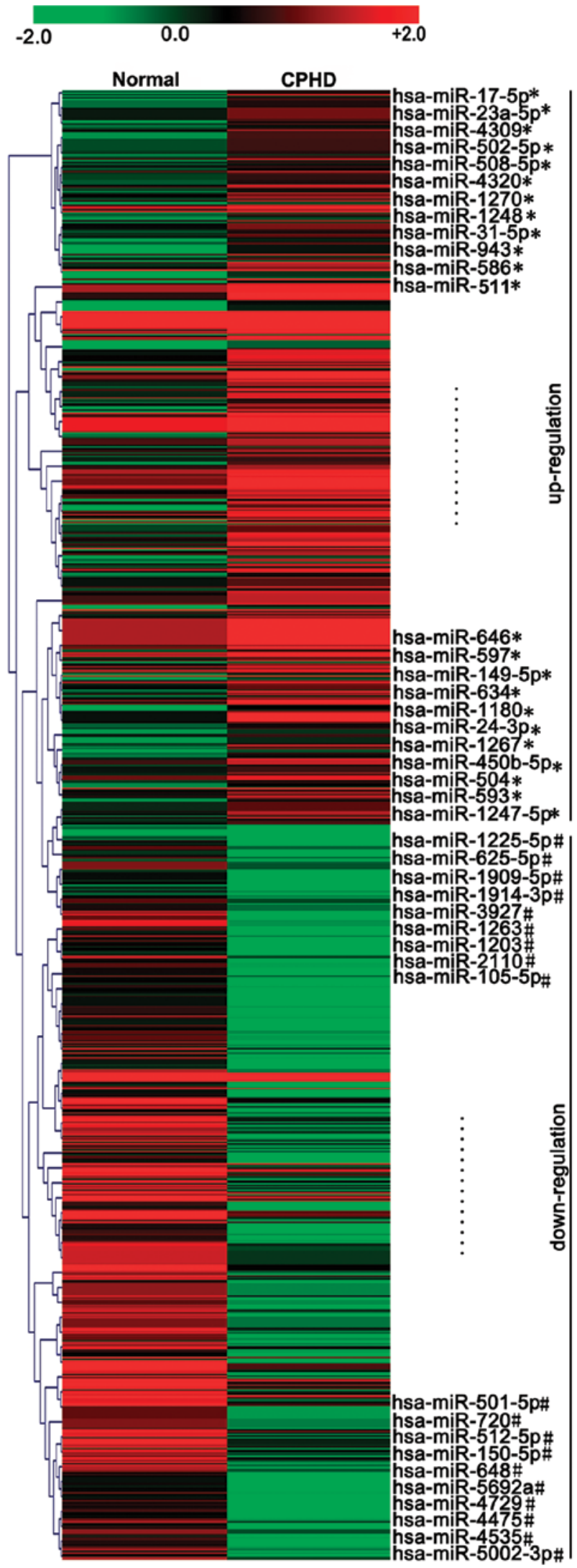

Figure 1. Heatmap and hierarchical clustering of miRNAs in serum of children with combined pituitary hormone deficiency (CPHD). The heatmap represents the results of the two-way hierarchical clustering of miRNAs in the serum of children with CPHD. Each row represents an miRNA, and each column represents samples tested. The clustering is represented for the miRNAs and samples on top. Red color represents miRNAs with an expression level above the mean value, and green color represents miRNAs with an expression level below the mean value. The 23 upregulated and 19 downregulated miRNAs identified are marked by an asterisk (*) or a hash (\#) symbol, respectively.
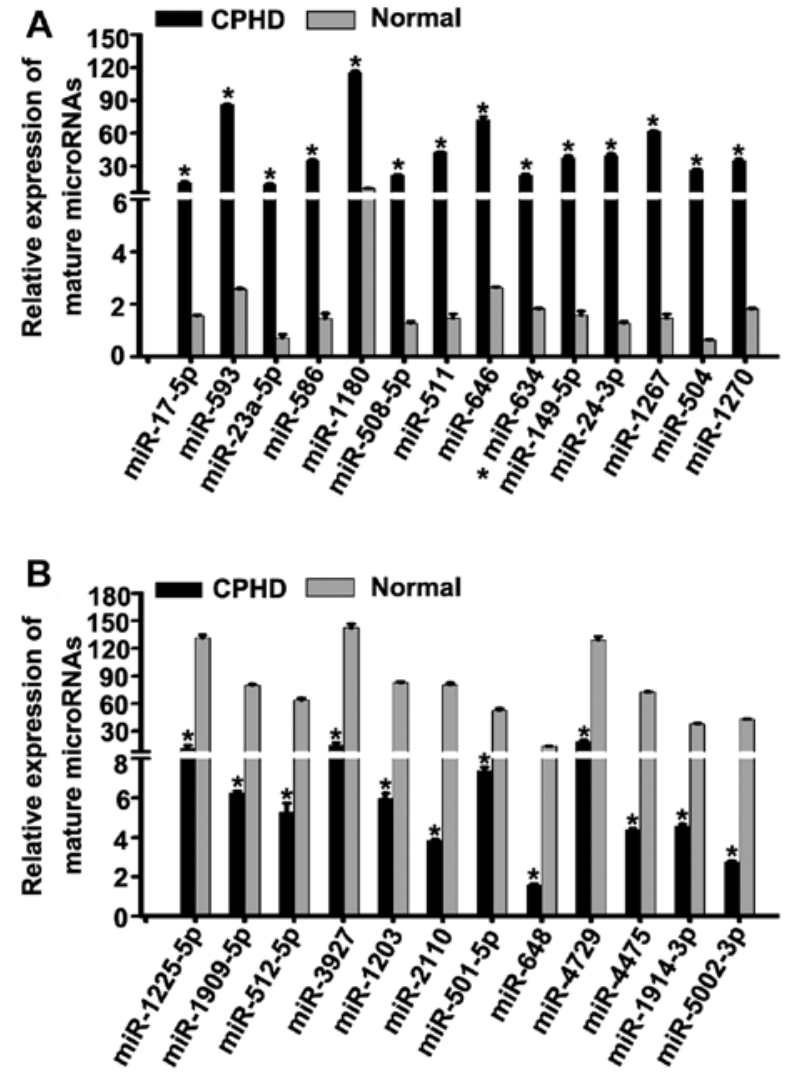

Figure 2. Identification of candidate miRNAs. (A) We detected the expression levels of 23 upregulated miNAs in children with combined pituitary hormone deficiency (CPHD) and normal (healthy) controls by RT-qPCR. The expression levels of miR-17-5p, miR-593, miR-23a-5p, miR-586, miR-1180, miR-508-5p, miR-511, miR-646, miR-634, miR-149-5p, miR-24-3p, miR-1267, miR-504 and miR-1270 were upregulated compared to the normal controls. (B) We examined the expression levels of 19 downregulated miRNAs in children with CPHD and normal controls by RT-qPCR. The expression levels of miR-1225-5p, miR1909-5p, miR-512-5p, miR-3927, miR-1203, miR-2110, miR-501-5p, miR-648, miR-4729, miR-4475, miR-1914-3p and miR-5002-5p were downregulated compared to the normal controls. * $\mathrm{P}<0.05$. Each bar represents the mean value \pm standard deviation from 3 independent experiments.

suggested the association of miR-593 and miR-511 with the PROP1 gene. The expression levels of miR-593 and miR-511 in the 103 children with CPHD and the normal controls were examined. The mean expression level of miR-593 was $149.06 \pm 72.34$ and that of miR-511 was $106.18 \pm 54.08$. The mean expression levels of miR-593 (149.06 \pm 72.34 vs. $34.89 \pm 24.61)$ and miR-511 (106.18 \pm 54.08 vs. $34.21 \pm 21.53)$ were upregulated compared to the normal controls (Fig. 3B; $\mathrm{P}<0.05$ ).

To verify the direct interaction between miR-593 and miR-511 and the 3'-UTR of the PROPl gene, we cloned the 3'-UTR region that was predicted to interact with miR-593 and miR-511 into a luciferase reporter vector (Fig. 4A). The HEK293T cells were transfected with the miR-593 precursor, miR-511 precursor, miR-593 inhibitor, miR-511 inhibitor or control oligonucleotides. As is shown in Fig. 4B, the upregulation of miR-593 and miR-511 inhibited PROP1 protein expression by approximately $87 \%$. The luciferase activity of the reporter plasmid with the wild-type 3'-UTR of the PROPl gene was markedly decreased in the cells transfected with the miR-593 precursor and miR-511 precursor compared to the luciferase activity of the reporter plasmid with the mutant 3 '-UTR of the $P R O P 1$ gene 
Table I. Differentially expressed miRNAs in children with CPHD with at least a 6-fold change in epxression selected by microarray data analysis.

\begin{tabular}{|c|c|c|c|c|}
\hline No. & Name & Corrected P-value & Fold change & Regulation \\
\hline 1 & hsa-miR-17-5p & 0.00038 & 9.737702 & Up \\
\hline 2 & hsa-miR-23a-5p & 0.00004531 & 7.1713095 & Up \\
\hline 3 & hsa-miR-4309 & 0.000814 & 13.075409 & Up \\
\hline 4 & hsa-miR-502-5p & 0.0000028 & 12.420118 & Up \\
\hline 5 & hsa-miR-508-5p & 0.000018 & 8.289489 & Up \\
\hline 6 & hsa-miR-4320 & 0.000029 & 7.698423 & Up \\
\hline 7 & hsa-miR-1270 & 0.00000478 & 7.7110386 & Up \\
\hline 8 & hsa-miR-1248 & 0.001356 & 16.126984 & Up \\
\hline 9 & hsa-miR-31-5p & 0.000029 & 8.6424675 & Up \\
\hline 10 & hsa-miR-943 & 0.00645 & 7.1091013 & Up \\
\hline 11 & hsa-miR-586 & 0.000781 & 9.900925 & Up \\
\hline 12 & hsa-miR-511 & 0.00000569 & 13.058901 & Up \\
\hline 13 & hsa-miR-646 & 0.0000056 & 6.3428288 & Up \\
\hline 14 & hsa-miR-597 & 0.0000848 & 8.018958 & Up \\
\hline 15 & hsa-miR-149-5p & 0.00264 & 8.6524935 & Up \\
\hline 16 & hsa-miR-634 & 0.000157 & 10.592684 & Up \\
\hline 17 & hsa-miR-1180 & 0.000457 & 11.971244 & Up \\
\hline 18 & hsa-miR-24-3p & 0.0000063 & 12.153815 & Up \\
\hline 19 & hsa-miR-1267 & 0.000487 & 6.773687 & Up \\
\hline 20 & hsa-miR-450b-5p & 0.000158 & 8.018958 & Up \\
\hline 21 & hsa-miR-504 & 0.0000056 & 6.3428288 & Up \\
\hline 22 & hsa-miR-593 & 0.000158 & 8.018958 & Up \\
\hline 23 & hsa-miR-1247-5p & 0.0000651 & 6.3428288 & Up \\
\hline 1 & hsa-miR-1225-5p & 0.000024 & -6.7412977 & Down \\
\hline 2 & hsa-miR-625-5p & 0.000467 & -12.856266 & Down \\
\hline 3 & hsa-miR-1909-5p & 0.000267 & -11.81031 & Down \\
\hline 4 & hsa-miR-1914-3p & 0.000984 & -8.549995 & Down \\
\hline 5 & hsa-miR-3927 & 0.000289 & -14.380777 & Down \\
\hline 6 & hsa-miR-1263 & 0.000451 & -9.568466 & Down \\
\hline 7 & hsa-miR-1203 & 0.000167 & -10.962637 & Down \\
\hline 8 & hsa-miR-2110 & 0.000287 & -11.463881 & Down \\
\hline 9 & hsa-miR-105-5p & 0.0000671 & -6.78255 & Down \\
\hline 10 & hsa-miR-501-5p & 0.0000205 & -8.172279 & Down \\
\hline 11 & hsa-miR-720 & 0.000091 & -10.1659565 & Down \\
\hline 12 & hsa-miR-512-5p & 0.00036 & -17.136076 & Down \\
\hline 13 & hsa-miR-150-5p & 0.000497 & -8.718422 & Down \\
\hline 14 & hsa-miR-648 & -7.745552 & -6.179926 & Down \\
\hline 15 & hsa-miR-5692 & -10.1659565 & -7.8253 & Down \\
\hline 16 & hsa-miR-4729 & -7.745552 & -13.084776 & Down \\
\hline 17 & hsa-miR-4475 & -6.1597967 & -9.571002 & Down \\
\hline 18 & hsa-miR-4535 & -7.383092 & -7.945036 & Down \\
\hline 19 & hsa-miR-5002-3p & -5.559328 & -10.245269 & Down \\
\hline
\end{tabular}

The threshold set for up- and downregulated microRNAs was a fold change $\geq 6.0$ and a P-value $\leq 0.05$. Our results found 23 upregulated and 19 downregulated microRNA abnormally expressed in children with CPHD compared with the normal (healthy) controls. miRNAs, microRNAs; CPHD, combined pituitary hormone deficiency.

(Fig. 4C; $\mathrm{P}<0.05$ ). Conversely, the luciferase activity of the reporter plasmid was not affected following transfection with
miR-593 inhibitor and miR-511 inhibitor compared to the antisense (AS)-miR-control (Fig. 4C) $(\mathrm{P}<0.05)$. 


\section{A}

5' ... AgGUgagcuacuggaAgagacAg... 3' UCUUUGgGGUCGUCUCUIGU

$5^{\prime}$

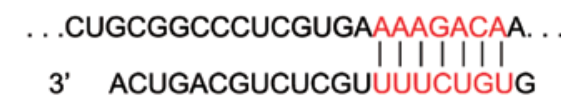

Position 49-56 of PROP1 3'UTR

hsa-miR-511

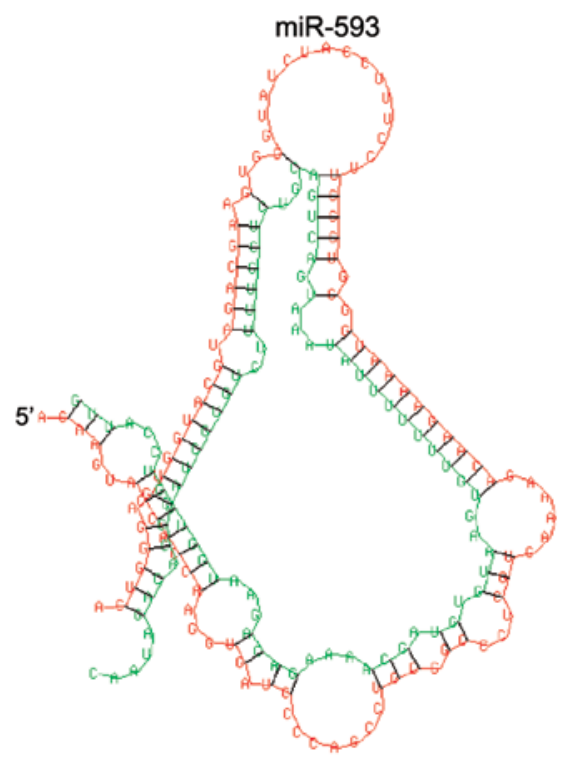

B

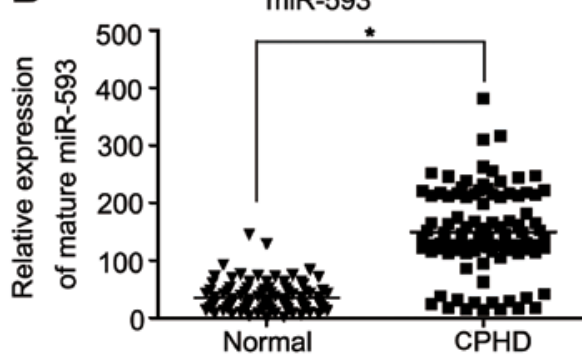

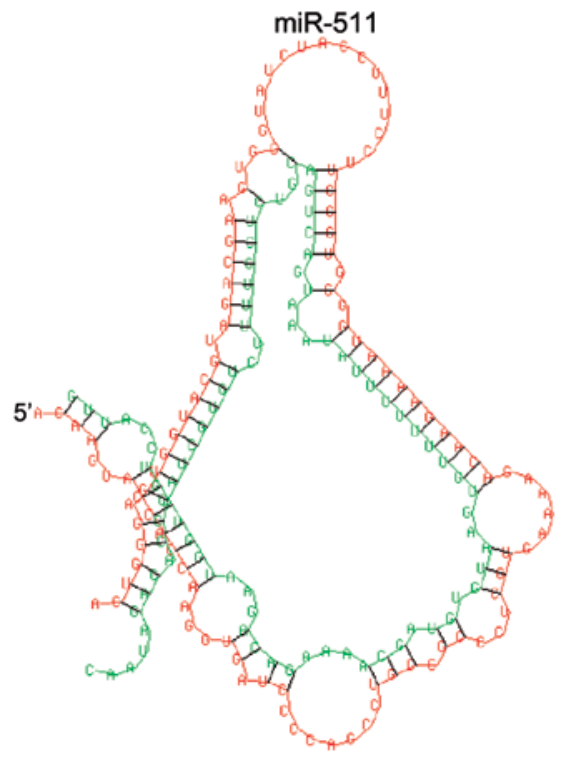

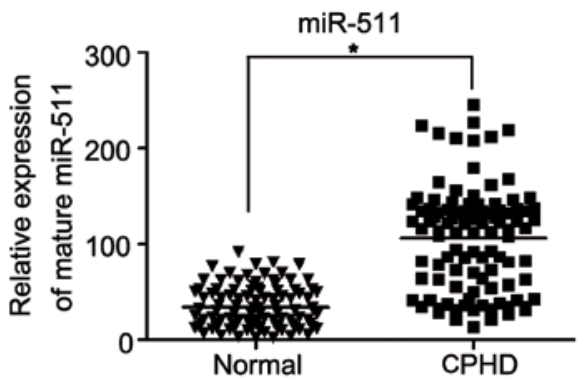

Figure 3. Candidate miRNAs, miR-593 and miR-511, were upregulated in children with combined pituitary hormone deficiency (CPHD) as shown by RT-qPCR. (A) We used several computational methods to predict the potential miR-593 and miR-511 targets using the microrna.org, TargetScan and RNAhybrid database online searching programs; only matched nucleotides with miRNA seed sequences are indicated with vertical lines. We identified that the PROP1 gene was a possible was a target of miR-593 and miR-511. (B) We examined the expression levels of miR-593 and miR-511 in the 103 children with CPHD and the 103 normal controls by RT-qPCR. The mean expression levels of miR-593 and miR-511 were upregulated compared with the normal controls. "P<0.05. Each bar represents the mean value \pm standard deviation from 3 independent experiments.

miR-593 and miR-511 as serum biomarkers for children with CPHD. The expression of miR-593 and miR-511 in the children with CPHD was significantly increased compared with the normal controls. The AUC was $0.912 \pm 0.020$ for miR-593 and was significantly higher than that of the null hypothesis (true area was 0.5) (Fig. 5A; $\mathrm{P}<0.01$ ). The AUC was $0.785 \pm 0.023$ for miR-511 and was significantly higher than that of the null hypothesis (true area was 0.5 ) (Fig. 5B; $\mathrm{P}<0.01$ ). According to Youden's index, the optimal operating point of the expression level of miR-593 and miR-511 in the serum was 108.27 and 63.43, respectively. At this cut-off point, the sensitivity was 82.54 and $84.86 \%$, and the specificity was 98.15 and $91.36 \%$ for miR-593 and miR-511, respectively.

\section{Discussion}

The pituitary gland produces hormones that play important roles in the development and the homeostasis of the body. A deficiency of two or more of these pituitary hormones, known as CPHD, may present in infants or children due to an unknown etiology and is considered congenital or idiopathic. To date, to the best of our knowledge, there is no study available on miRNAs in CPHD. miRNAs are a class of small non-coding RNAs and have been discovered in animals and plants. miRNAs are 19-22 nucleotide non-coding RNAs and are able to bind complementary sequences in the 3'-UTR of target mRNAs to induce their degradation or translational repression (24-26). They are encoded by genes that are presumably transcribed into single or clustered primary transcripts, which are processed and produce mature miRNAs. They function as regulators of disease initiation, progression and metastasis. miRNAs are a novel class of regulatory molecules with the ability to control gene expression at the post-transcriptional level. They appear decrease protein expression by blocking the translation of mRNAs into proteins. Therefore, the identification of CPHDspecific miRNAs is critical for understanding their role in the 

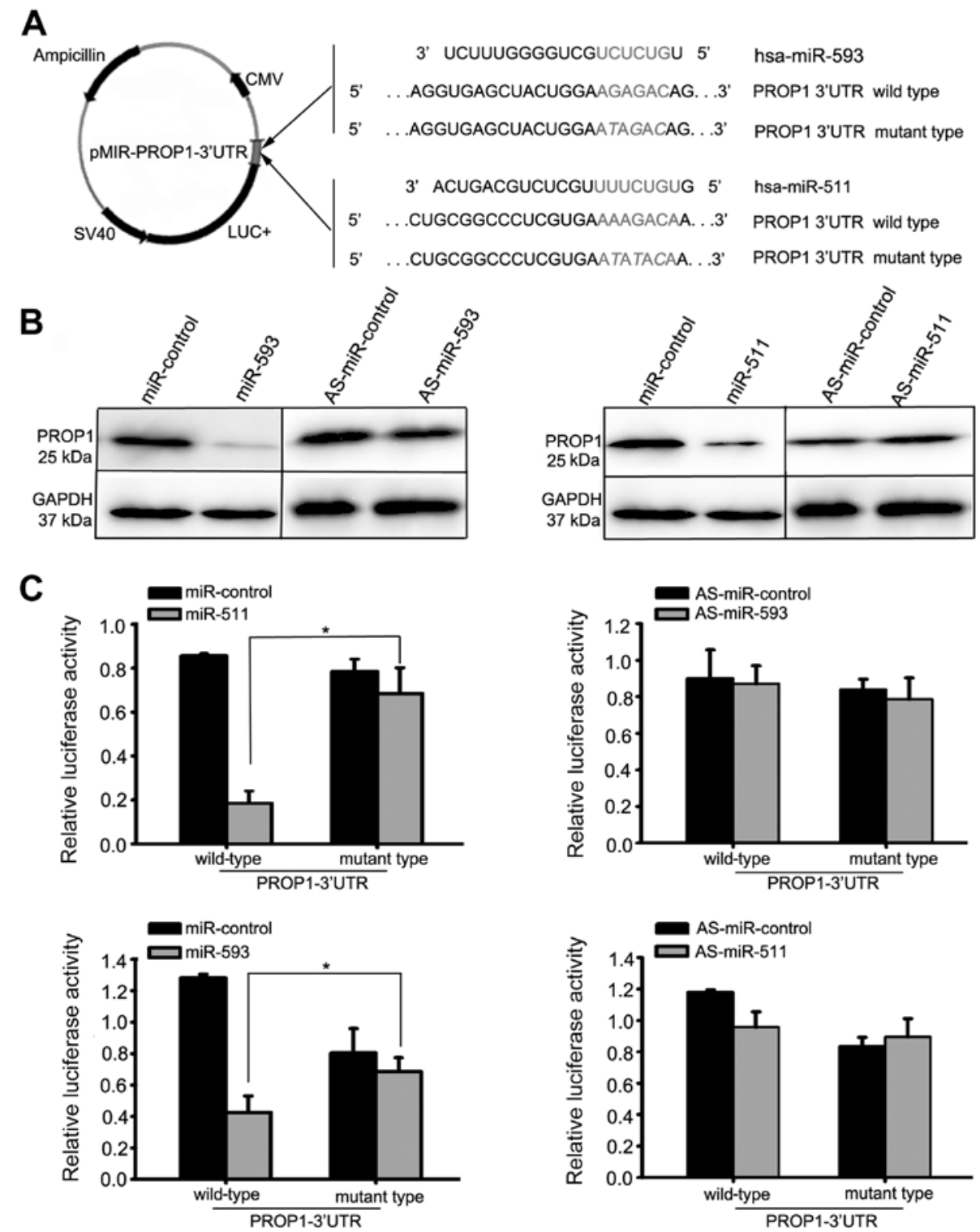

Figure 4. miR-593 and miR-511 target the 3'-untranslated regions (3'-UTR) of the PROP1 gene and decrease PROP1 expression. (A) Representative nucleotide sequence matches between possible target sequences and miRNAs. The miR-593 seed sequence (UCUCUG) and miR-511 seed sequence (UUUCUGU) is shown (gray and italic nucleotides). Schematic graph of the 3'-UTR binding site for miR-593 or miR-511. PROPI-3'-UTR-wild-type or PROPI-3'-UT-mutant type was inserted downstream of the luciferase of pMIR-reporter vector. (B) At $48 \mathrm{~h}$ after the transfection of miR-593 precursor, miR-511 precursor, miR-593 inhibitor, miR-511 inhibitor or control oligonucleotides into the HEK293T cells, the PROP1 protein level was significantly reduced in the cells transfected with miR-593 precursor or miR-511 precursor, as shown by western blot analysis. By contrast, transfection with miR-593 inhibitor or miR-511 inhibitor did not affect the protein expression of PROP1. (C) We assessed the luciferase activity by co-transfecting the luciferase reporter vector bearing the 3'-UTR of the PROPI gene with the miR-593 precursor, miR-511 precursor, miR-593 inhibitor, miR-511 inhibitor or control oligonucleotides. Luciferase activity of reporter plasmid with wild-type 3'-UTR of the PROPI gene was markedly decreased in the cells transfected with miR-593 precursor and miR-511 precursor, compared with the luciferase activity of the reporter plasmid with mutant 3'-UTR of PROP1. Each bar represents the mean values \pm SD from 3 independent experiments ("P $<0.05$ ).
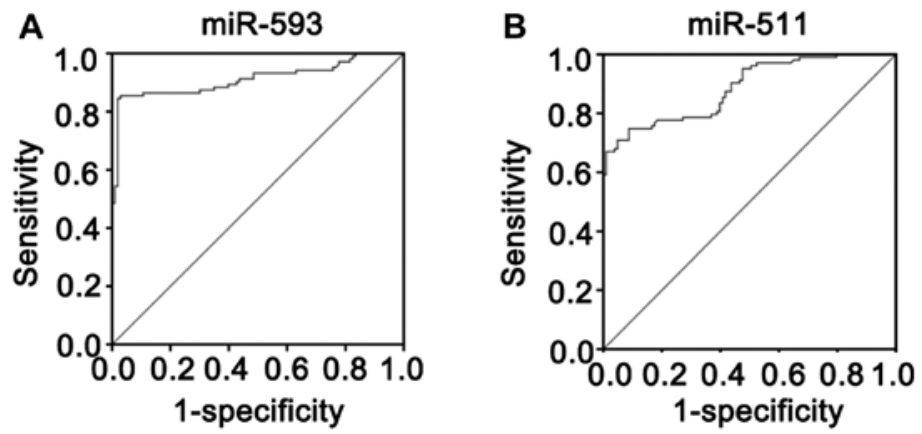

Figure 5. miR-593 and miR-511 may serve as serum biomarkers for children with combined pituitary hormone deficiency (CPHD). (A) The ROC curve of the serum of the 103 children with CPHD and the 103 normal controls for miR-593. The AUC was $0.912 \pm 0.02$ for miR-593, with a sensitivity and specificity of 82.54 and $98.15 \%$, respectively. (B) The ROC curve of the serum of the 103 children with CPHD and the 103 normal controls for miR-511. The AUC was $0.785 \pm 0.023$ for miR-511, with a sensitivity and specificity of 84.86 and $91.36 \%$, respectively. 
pathophysiological basis of CPHD and may prove useful for finding novel therapeutic methods. Intriguingly, it has been suggested that miRNAs are present in human peripheral blood in a consistent, reproducible and stable manner (19). More importantly, a series of studies have demonstrated that at least in some pathological conditions, such as cancer, heart failure and liver damage, circulating miRNAs may in part reflect tissue damage $(13,14,27,28)$. This discovery opens up the possibility of using miRNAs as non-invasive biomarkers for CPHD.

Hitherto, there data on the association between miRNAs and CPHD are limited. Microarray analysis is a powerful technology that is able to perform genome-wide analysis in one experiment. miRNA expression profiles can characterize miRNAs that are differentially regulated under different experimental conditions. Thus, in this study, we used a global miRNA microarray to identify specific miRNAs in CPHD. Our results revealed 23 upregulated and 19 downregulated miRNAs that were abnormally expressed in children with CPHD compared with the normal controls. The expression levels of miR-17-5p, miR-593, miR-23a-5p, miR-586, miR-1180, miR-508-5p, miR-511, miR-646, miR-634, miR-149-5p, miR-24-3p, miR-1267, miR-504 and miR-1270 were upregulated. The expression levels of miR-1225-5p, miR-1909-5p, miR-512-5p, miR-3927, miR-1203, miR-2110, miR-501-5p, miR-648, miR-4729, miR-4475, miR-1914-3p and miR-5002-5p were downregulated in the children with CPHD. These data indicate that the characteristics of serum miRNA expression are associated with children with CPHD and provide a valuable repertoire that can be used to discover circulating miRNA-based biomarkers for the diagnosis of CPHD. To better understand the role of specific miRNAs in the diagnosis of CPHD, the microrna.org, TargetScan and RNAhybrid database online searching programs were used to predict putative targets. We identified that the PROPI gene was a possible target of miR-593 and miR-511; further experimental procedures were carried out to validate these findings. Our results revealed that the expression levels of miR-593 or miR-511 in the serum of children with CPHD were upregulated compared with the normal controls. miR-593 and miR-511 directly targeted the PROPI gene and this was confirmed by western blot analysis and luciferase activity assay following the transfection of HEK293T cells with miR-593 precursor, miR-511 precursor, miR-593 inhibitor, miR-511 inhibitor or control oligonucleotides. The above findings support the hypothesis that miRNAs may be involved in the development of CPHD. In clinical practice, the tissue in children with CPHD is not readily accessible. The ROC curve and the AUC for the expression levels of the serum of the 103 children with CPHD and the 103 normal controls were calculated. The AUC was $0.912 \pm 0.020$ for miR-593 and the AUC was $0.785 \pm 0.023$ for miR-511. Thus, the expression levels of miR-593 and miR-511 in serum may serve as a molecular marker for children with CPHD. To identify an optimal cut-off point to detect children with CPHD, Youden's index was used in this study. According to Youden's index, the optimal operating point of the blood expression level of miR-593 and miR-511 was 108.27 and 63.43, respectively. At this cut-off point, the sensitivity was 82.54 and $84.86 \%$, specificity was 98.15 and $91.36 \%$ for miR-593 and miR-511, respectively. Taken together, these data suggest that miR-593 and miR-511 directly target the PROPl gene and may serve as serum biomarkers for children with CPHD.

In conclusion, in the present study, we found that the levels of miR-593 and miR-511 in the serum of children with CPHD were significantly increased and that these miRNAs directly targeted the PROPI gene. This suggests that circulating levels of miR-593 or miR-511 may serve as novel biomarkers for the clinical diagnosis of CPHD. Thus, miRNAs cannot be overlooked as a class of molecules that regulate biological functions and CPHD. Our data broaden the understanding of the functions of miRNAs in children with CPHD. Considering the small sample size used in the present study, investigations including a larger scale of patients are warranted. Additionally, further studies are required to reveal the exact time course of miR-593 and miR-511 in the serum of children with CPHD. Although circulating miRNA levels can be detected by real-time PCR, the underlying mechanisms responsible for the increased circulating miRNA levels and whether they have pathophysiological functions in CPHD require further investigation.

\section{Acknowledgements}

We would like to thank Professor Qiaoming Zhi for providing technical support with the in vitro experiments. We are grateful to our patients and the families who consented to be a part of this study. This study was supported in part by grants from the National Youthful Science Foundation of China (no. 81101858), and the Shandong Science and Technology Commission of China (no. 2013GSF11817).

\section{References}

1. Kandemir N, Vurallı D, Taşkıran E, Gönç N, Özön A, Alikasifoğlu A and Yilmaz E: Frequency of mutations in PROP-1 gene in Turkish children with combined pituitary hormone deficiency. Turk J Pediatr 54: 570-575, 2012.

2. Kelberman D, Turton JP, Woods KS, et al: Molecular analysis of novel PROP1 mutations associated with combined pituitary hormone deficiency (CPHD). Clin Endocrinol (Oxf) 70: 96-103, 2009.

3. Reynaud R, Albarel F, Saveanu A, et al: Pituitary stalk interruption syndrome in 83 patients: novel HESX1 mutation and severe hormonal prognosis in malformative forms. Eur J Endocrinol 164: 457-465, 2011.

4. Tauber M, Chevrel J, Diene G, et al: Long-term evolution of endocrine disorders and effect of GH therapy in 35 patients with pituitary stalk interruption syndrome. Horm Res 64: 266-273, 2005.

5. Cruz JB, Nunes VS, Clara SA, Perone D, Kopp P and Nogueira CR: Molecular analysis of the PROP1 and HESX1 genes in patients with septo-optic dysplasia and/or pituitary hormone deficiency. Arq Bras Endocrinol Metabol 54: 482-487, 2010.

6. Ward RD, Raetzman LT, Suh H, Stone BM, Nasonkin IO and Camper SA: Role of PROP1 in pituitary gland growth. Mol Endocrinol 19: 698-710, 2005.

7. Cogan JD, Wu W, Phillips JA III, et al: The PROP1 2-base pair deletion is a common cause of combined pituitary hormone deficiency. J Clin Endocrinol Metab 83: 3346-3349, 1998.

8. Cohen LE, Wondisford FE, Salvatoni A, Maghnie M, Brucker-Davis F, Weintraub BD and Radovick S: A 'hot spot' in the Pit-1 gene responsible for combined pituitary hormone deficiency: clinical and molecular correlates. J Clin Endocrinol Metab 80: 679-684, 1995.

9. Wu W, Cogan JD, Pfaffle RW, et al: Mutations in PROP1 cause familial combined pituitary hormone deficiency. Nat Genet 18: 147-149, 1998.

10. Arroyo A, Pernasetti F, Vasilyev VV, Amato P, Yen SS and Mellon PL: A unique case of combined pituitary hormone deficiency caused by a PROP1 gene mutation (R120C) associated with normal height and absent puberty. Clin Endocrinol (Oxf) 57: 283-291, 2002. 
11. Deladoëy J1, Flück C, Büyükgebiz A, et al: 'Hot spot' in the PROP1 gene responsible for combined pituitary hormone deficiency. J Clin Endocrinol Metab 84: 1645-1650, 1999.

12. Parks JS, Brown MR, Hurley DL, Phelps CJ and Wajnrajch MP Heritable disorders of pituitary development. J Clin Endocrinol Metab 84: 4362-4370, 1999.

13. Heneghan HM, Miller N, Lowery AJ, Sweeney KJ, Newell J and Kerin MJ: Circulating microRNAs as novel minimally invasive biomarkers for breast cancer. Ann Surg 251: 499-505, 2010.

14. Tijsen AJ, Creemers EE, Moerland PD, de Windt LJ, van der Wal AC, Kok WE and Pinto YM: MiR423-5p as a circulating biomarker for heart failure. Circ Res 106: 1035-1039, 2010.

15. Vlassov VV, Laktionov PP and Rykova EY: Circulating nucleic acids as a potential source for cancer biomarkers. Curr Mol Med 10: $142-165,2010$

16. Cortez MA and Calin GA: MicroRNA identification in plasma and serum: a new tool to diagnose and monitor diseases. Expert Opin Biol Ther 9: 703-711, 2009.

17. Hoekstra M, van der Lans CA, Halvorsen B, et al: The peripheral blood mononuclear cell microRNA signature of coronary artery disease. Biochem Biophys Res Commun 394: 792-797, 2010.

18. Tan KS, Armugam A, Sepramaniam S, Lim KY, Setyowati KD, Wang CW and Jeyaseelan K: Expression profile of MicroRNAs in young stroke patients. PLoS One 4: e7689, 2009.

19. Chen X, Ba Y, Ma L, et al: Characterization of microRNAs in serum: a novel class of biomarkers for diagnosis of cancer and other diseases. Cell Res 18: 997-1006, 2008.

20. Zampetaki A, Kiechl S, Drozdov I, et al: Plasma microRNA profiling reveals loss of endothelial miR-126 and other microRNAs in type 2 diabetes. Circ Res 107: 810-817, 2010.
21. Guo X, Guo L, Ji J, et al: miRNA-331-3p directly targets E2F1 and induces growth arrest in human gastric cancer. Biochem Biophys Res Commun 398: 1-6, 2010.

22. Ratert N, Meyer HA, Jung M, et al: miRNA profiling identifies candidate mirnas for bladder cancer diagnosis and clinical outcome. J Mol Diagn 15: 695-705, 2013.

23. Sethi S, Kong D, Land S, Dyson G, Sakr WA and Sarkar FH: Comprehensive molecular oncogenomic profiling and miRNA analysis of prostate cancer. Am J Transl Res 5: 200-211, 2013.

24. Lagos-Quintana M, Rauhut R, Lendeckel W and Tuschl T: Identification of novel genes coding for small expressed RNAs. Science 294: 853-858, 2001.

25. Lau NC, Lim LP, Weinstein EG and Bartel DP: An abundant class of tiny RNAs with probable regulatory roles in Caenorhabditis elegans. Science 294: 858-862, 2001.

26. Lee RC and Ambros V: An extensive class of small RNAs in Caenorhabditis elegans. Science 294: 862-864, 2001.

27. Si H, Sun X, Chen Y, Cao Y, Chen S, Wang H and Hu C: Circulating microRNA-92a and microRNA-21 as novel minimally invasive biomarkers for primary breast cancer. J Cancer Res Clin Oncol 139: 223-229, 2013.

28. Wang K, Zhang S, Marzolf B, et al: Circulating microRNAs, potential biomarkers for drug-induced liver injury. Proc Natl Acad Sci USA 106: 4402-4407, 2009.

29. Wang GK, Zhu JQ, Zhang JT, et al: Circulating microRNA: a novel potential biomarker for early diagnosis of acute myocardial infarction in humans. Eur Heart J 31: 659-666, 2010. 